\title{
Nonlocal Integrodifferential Boundary Value Problem for Nonlinear Fractional Differential Equations on an Unbounded Domain
}

\author{
Lihong Zhang, ${ }^{1}$ Bashir Ahmad, ${ }^{2}$ Guotao Wang, ${ }^{1}$ Ravi P. Agarwal, ${ }^{2,3}$ \\ Maryem Al-Yami, ${ }^{2}$ and Wafa Shammakh ${ }^{2}$ \\ ${ }^{1}$ School of Mathematics and Computer Science, Shanxi Normal University, Linfen, Shanxi 041004, China \\ ${ }^{2}$ Department of Mathematics, Faculty of Science, King Abdulaziz University, P.O. Box 80203, Jeddah 21589, Saudi Arabia \\ ${ }^{3}$ Department of Mathematics, Texas A and M University, Kingsville, TX 78363-8202, USA
}

Correspondence should be addressed to Bashir Ahmad; bashirahmad_qau@yahoo.com

Received 8 May 2013; Accepted 10 June 2013

Academic Editor: Dumitru Baleanu

Copyright (c) 2013 Lihong Zhang et al. This is an open access article distributed under the Creative Commons Attribution License, which permits unrestricted use, distribution, and reproduction in any medium, provided the original work is properly cited.

\begin{abstract}
This paper investigates the existence of nonnegative solutions for nonlinear fractional differential equations with nonlocal fractional integrodifferential boundary conditions on an unbounded domain by means of Leray-Schauder nonlinear alternative theorem. An example is discussed for the illustration of the main work.
\end{abstract}

\section{Introduction}

Recent studies on fractional differential equations, appeared in several special issues and books, reveal an extensive development of various aspects of the subject. One of the reasons for the popularity of fractional calculus is the nonlocal behavior of fractional-order operators in contrast to the classical integer-order operators. This characteristic has motivated many experts on modelling to introduce the concept of fractional modelling by taking into account the ideas of fractional calculus. Examples include various disciplines of science and engineering such as physics, chemistry, biomathematics, dynamical processes in porous media, dynamics of earthquakes, material viscoelastic theory, and control theory of dynamical systems. Furthermore, the outcome of certain experimentations indicate that integral and derivative operators of fractional order possess some characteristics related to complex systems having long memory in time. For details and examples, we refer the reader to the works in [1-7].

Boundary value problems of fractional-order differential equations have been extensively investigated during the last few years, and a variety of results on the topic have been established. A great deal of the work on fractional boundary value problems involves local/nonlocal boundary conditions on bounded and unbounded domains; for example, see [8-26].

In this paper, we study a new class of problems on fractional differential equations with nonlocal boundary conditions on unbounded domains. Precisely, we consider the following problem:

$$
\begin{aligned}
D^{\alpha} u(t)+f(t, u(t))= & 0, \quad 1<\alpha \leq 2, t \in J=[0,+\infty), \\
& I^{2-\alpha} u(0)=0, \\
D^{\alpha-1} u(+\infty)= & \lambda I^{\alpha-1} u(\eta), \quad 0<\lambda, \eta<\infty,
\end{aligned}
$$

where $D^{\alpha}$ denotes Riemann-Liouville fractional derivative of order $\alpha, f \in C\left(J \times \mathbb{R}, \mathbb{R}^{+}\right)$, and $\mathbb{R}^{+}=[0,+\infty)$.

\section{Preliminaries}

In this section, we present some useful definitions and related theorems. 
Definition 1 (see [4]). The Riemann-Liouville fractional derivative of order $\delta$ for a continuous function $f$ is defined by

$$
\begin{array}{r}
D^{\delta} f(t)=\frac{1}{\Gamma(n-\delta)}\left(\frac{d}{d t}\right)^{n} \int_{0}^{t}(t-s)^{n-\delta-1} f(s) d s, \\
n=[\delta]+1,
\end{array}
$$

Provided that the right hand side is pointwise defined on $(0, \infty)$ and $[\delta]$ is the integer part of $\delta$.

Definition 2 (see [4]). The Riemann-Liouville fractional integral of order $\delta$ for a function $f$ is defined as

$$
I^{\delta} f(t)=\frac{1}{\Gamma(\delta)} \int_{0}^{t}(t-s)^{\delta-1} f(s) d s, \quad \delta>0,
$$

provided that such integral exists.

Theorem 3 (see [27] (Leray-Schauder nonlinear alternative)). Let $C$ be a convex subset of a Banach space, and let $U$ be an open subset of $C$ with $0 \in U$. Then every completely continuous map $N: \bar{U} \rightarrow C$ has at least one of the following two properties:

(1) $N$ has a fixed point in $\bar{U}$;

(2) there is an $x \in \partial U$ and $\lambda \in(0,1)$ with $x=\lambda N x$.

Theorem 4 (see [28]). Let $U \subset X$ be a bounded set. Then $U$ is relatively compact in $X$ if the following conditions hold:

(i) for any $u(t) \in U, u(t) /\left(1+t^{\alpha-1}\right)$ is equicontinuous on any compact interval of $J$;

(ii) for any $\varepsilon>0$, there exists a constant $T=T(\varepsilon)>0$ such that

$$
\left|\frac{u\left(t_{1}\right)}{1+t_{1}^{\alpha-1}}-\frac{u\left(t_{2}\right)}{1+t_{2}^{\alpha-1}}\right|<\varepsilon
$$

for any $t_{1}, t_{2} \geq T$ and $u \in U$.

Now we list the assumptions needed in the sequel.

$\left(H_{1}\right): \Gamma(2 \alpha-1)>\lambda \eta^{2 \alpha-2}$.

$\left(H_{2}\right)$ : there exist nonnegative functions $a(t), b(t)$ defined on

$[0, \infty)$ and a constant $\rho>0$ such that

$$
\begin{gathered}
|f(t, u(t))| \leq a(t)+b(t)|u(t)|^{\rho}, \\
\int_{0}^{+\infty} a(t) d t=a^{*}<+\infty, \\
\int_{0}^{+\infty} b(t)\left(1+t^{\alpha-1}\right)^{\rho} d t=b^{*}<+\infty .
\end{gathered}
$$

\section{Some Lemmas}

This section contains some preliminary works that we need to establish the main result for problem (1).
Lemma 5. Let $\sigma(t) \in C([0,+\infty))$ with $\int_{0}^{\infty} \sigma(s) d s<\infty$. For $\Gamma(2 \alpha-1) \neq \lambda \eta^{2 \alpha-2}$, the associated linear fractional boundary value problem,

$$
\begin{gathered}
D^{\alpha} u(t)+\sigma(t)=0, \quad 1<\alpha \leq 2, \\
I^{2-\alpha} u(0)=0, \\
D^{\alpha-1} u(+\infty)=\lambda I^{\alpha-1} u(\eta),
\end{gathered}
$$

has a unique solution given by

$$
u(t)=\int_{0}^{+\infty} G(t, s) \sigma(s) d s
$$

where

$$
\begin{aligned}
& G(t, s)
\end{aligned}
$$

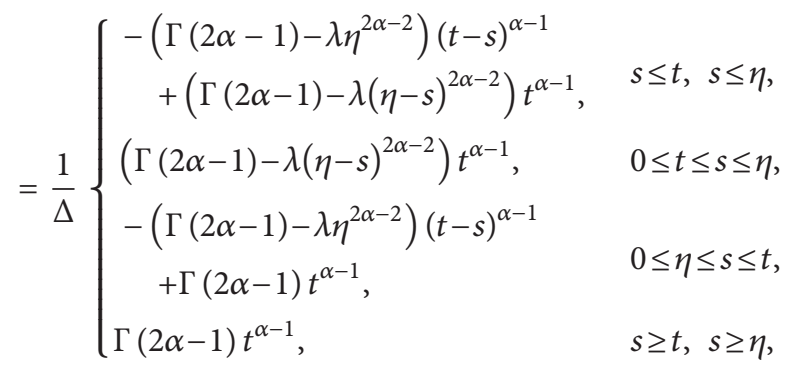

$$
\Delta=\Gamma(\alpha)\left(\Gamma(2 \alpha-1)-\lambda \eta^{2 \alpha-2}\right) .
$$

Proof. It is well known that the fractional equation in (6) is equivalent to the integral equation:

$$
u(t)=-I^{\alpha} \sigma(t)+c_{1} t^{\alpha-1}+c_{0} t^{\alpha-2},
$$

where $c_{0}, c_{1} \in \mathbb{R}$ are arbitrary constants. From (10), we have

$$
\begin{gathered}
D^{\alpha-1} u(t)=-I^{1} \sigma(t)+c_{1} \Gamma(\alpha)=c_{1} \Gamma(\alpha)-\int_{0}^{t} \sigma(s) d s \\
D^{\alpha-2} u(t)=-I^{2} \sigma(t)+c_{1} \Gamma(\alpha-1) t+c_{0} \Gamma(\alpha-1) .
\end{gathered}
$$

Using the given boundary conditions in (10), we find that $c_{0}=0$ and

$$
\begin{aligned}
c_{1}= & \frac{1}{\Gamma(\alpha)-A} \int_{0}^{\infty} \sigma(s) d s-\frac{\lambda}{\Gamma(\alpha)-A} \\
& \times \int_{0}^{\eta} \frac{(\eta-s)^{\alpha-2}}{\Gamma(\alpha-1)}\left[\int_{0}^{s} \frac{(s-x)^{\alpha-1}}{\Gamma(\alpha)} \sigma(x) d x\right] d s \\
= & \frac{1}{\Gamma(\alpha)-A} \int_{0}^{\infty} \sigma(s) d s-\frac{\lambda}{\Gamma(\alpha)-A} I^{2 \alpha-1} \sigma(\eta),
\end{aligned}
$$

where

$$
A=\lambda \int_{0}^{\eta} \frac{s^{\alpha-1}(\eta-s)^{\alpha-2}}{\Gamma(\alpha-1)} d s=\frac{\lambda \Gamma(\alpha) \eta^{2 \alpha-2}}{\Gamma(2 \alpha-1)} .
$$


Substituting the values of $c_{1}, c_{2}$ into (10) gives

$$
\begin{aligned}
u(t)= & -\int_{0}^{t} \frac{(t-s)^{\alpha-1}}{\Gamma(\alpha)} \sigma(s) d s-\frac{\lambda t^{\alpha-1}}{\Gamma(\alpha)-A} I^{2 \alpha-1} \sigma(\eta) \\
& \quad+\frac{t^{\alpha-1}}{\Gamma(\alpha)-A} \int_{0}^{\infty} \sigma(s) d s \\
= & -\int_{0}^{t} \frac{(t-s)^{\alpha-1}}{\Gamma(\alpha)} \sigma(s) d s-\frac{\Gamma(2 \alpha-1) \lambda t^{\alpha-1}}{\Gamma(\alpha)\left(\Gamma(2 \alpha-1)-\lambda \eta^{2 \alpha-2}\right)} \\
& \times \int_{0}^{\eta} \frac{(\eta-s)^{2 \alpha-2}}{\Gamma(2 \alpha-1)} \sigma(s) d s+\frac{\Gamma(2 \alpha-1) t^{\alpha-1}}{\Gamma(\alpha)\left(\Gamma(2 \alpha-1)-\lambda \eta^{2 \alpha-2}\right)} \\
& \times \int_{0}^{\infty} \sigma(s) d s \\
= & \int_{0}^{\infty} G(t, s) \sigma(s) d s,
\end{aligned}
$$

where $G(t, s)$ is defined by (8).

Remark 6. In view of the assumption $\left(H_{1}\right)$, Green's function $G(t, s)$ satisfies the properties:

(1) $G(t, s) \geq 0$,

(2)

$$
\frac{G(t, s)}{1+t^{\alpha-1}} \leq \frac{\Gamma(2 \alpha-1)}{\Gamma(\alpha)\left[\Gamma(2 \alpha-1)-\lambda \eta^{2 \alpha-2}\right]} \triangleq L .
$$

For the forthcoming analysis, we introduce a space

$$
X=\left\{u \in C(J, \mathbb{R}): \sup _{t \in J} \frac{|u(t)|}{1+t^{\alpha-1}}<+\infty\right\},
$$

equipped with the norm

$$
\|u\|_{X}=\sup _{t \in J} \frac{|u(t)|}{1+t^{\alpha-1}}
$$

Notice that $X$ is a Banach space.

Define an operator $T: X \rightarrow X$ as follows:

$$
T u(t)=\int_{0}^{\infty} G(t, s) f(s, u(s)) d s
$$

Observe that problem (1) has a solution only if the operator $T$ has a fixed point.

Lemma 7. If $\left(H_{1}\right),\left(H_{2}\right)$ hold, then the operator $T: X \rightarrow X$ is completely continuous.

Proof. We divide the proof into several steps. (i) The operator $T: X \rightarrow X$ is uniformly bounded. Let $\Omega$ be any bounded subset of $X$; then there exists a constant $L_{1}$ such that $\|u\|_{X} \leq L_{1}$. By $\left(H_{2}\right)$, we have

$$
\begin{aligned}
\|T u\|_{X} & =\sup _{t \in J} \int_{0}^{\infty} \frac{G(t, s)}{1+t^{\alpha-1}}|f(s, u(s))| d s \\
& \leq L \int_{0}^{\infty}\left[a(s)+b(s)\left(1+s^{\alpha-1}\right)^{\rho} \frac{|u(s)|^{\rho}}{\left(1+s^{\alpha-1}\right)^{\rho}}\right] d s \\
& \leq L\left(a^{*}+b^{*} L_{1}^{\rho}\right) \\
& <\infty .
\end{aligned}
$$

This shows that $T \Omega$ is uniformly bounded.

(ii) $T: X \rightarrow X$ is continuous. Take $u_{n}, u \in X$ such that $\left\|u_{n}\right\|_{X}<\infty,\|u\|_{X}<\infty$, and $u_{n} \rightarrow u$ as $n \rightarrow \infty$. Then, by $\left(H_{2}\right)$, we have

$$
\begin{aligned}
\int_{0}^{\infty} \frac{G(t, s)}{1+t^{\alpha-1}} f\left(s, u_{n}(s)\right) d s & \leq L \int_{0}^{\infty}\left[a(s)+b(s)\left|u_{n}(s)\right|^{\rho}\right] d s \\
& \leq L a^{*}+L b^{*}\left\|u_{n}\right\|_{X}^{\rho}<\infty
\end{aligned}
$$

where $L$ is defined by (15).

By the Lebesgue dominated convergence theorem and continuity of $f$, we obtain

$\lim _{n \rightarrow \infty} \int_{0}^{\infty} \frac{G(t, s)}{1+t^{\alpha-1}} f\left(s, u_{n}(s)\right) d s=\int_{0}^{\infty} \frac{G(t, s)}{1+t^{\alpha-1}} f(s, u(s)) d s$.

Taking the limit $n \rightarrow \infty$, we get

$$
\begin{aligned}
\left\|T u_{n}-T u\right\|_{X}= & \sup _{t \in J} \int_{0}^{\infty} \frac{G(t, s)}{1+t^{\alpha-1}} \\
& \times\left|f\left(s, u_{n}(s)\right)-f(s, u(s))\right| d s \longrightarrow 0 .
\end{aligned}
$$

Therefore, $T$ is continuous.

(iii) $T: X \rightarrow X$ is equicontinuous. We consider two cases.

(a) Let $I \subset J$ be any compact interval, and let $t_{1}, t_{2} \in I$ be such that $t_{1}<t_{2}$. Let $\Omega$ be any bounded subset of $X$; then for any $u \in \Omega$, we have

$$
\begin{aligned}
& \left|\frac{T u\left(t_{2}\right)}{1+t_{2}^{\alpha-1}}-\frac{T u\left(t_{1}\right)}{1+t_{1}^{\alpha-1}}\right| \\
& \quad=\left|\int_{0}^{\infty}\left(\frac{G\left(t_{2}, s\right)}{1+t_{2}^{\alpha-1}}-\frac{G\left(t_{1}, s\right)}{1+t_{1}^{\alpha-1}}\right) f(s, u(s)) d s\right| \\
& \quad \leq \int_{0}^{\infty}\left|\frac{G\left(t_{2}, s\right)}{1+t_{2}^{\alpha-1}}-\frac{G\left(t_{1}, s\right)}{1+t_{1}^{\alpha-1}}\right|\left(a(s)+b(s)\left(1+s^{\alpha-1}\right)^{\rho}\|u\|_{X}^{\rho}\right) d s .
\end{aligned}
$$

Since $G(t, s)$ is continuous on $J \times J$, we have that $G(t, s) /(1+$ $\left.t^{\alpha-1}\right)$ is a uniformly continuous function on the compact set 
$I \times I$. Moreover, for $s \geq t$, we have that this function only depends on $t$; in consequence it is uniformly continuous on $I \times(J \backslash I)$. So we have that for all $s \in J$ and $t_{1}, t_{2} \in I$, the following property holds.

For all $\varepsilon>0$ there is $\delta(\varepsilon)>0$ such that if $\left|t_{1}-t_{2}\right|<\delta$, then $\left|G\left(t_{2}, s\right) /\left(1+t_{2}^{\alpha-1}\right)-G\left(t_{1}, s\right) /\left(1+t_{1}^{\alpha-1}\right)\right|<\varepsilon$. By this, together with (23), and the fact that

$$
\int_{0}^{\infty}\left(a(s)+b(s)\left(1+s^{\alpha-1}\right)^{\rho} L_{1}\right) d s<\infty,
$$

we can get that $T \Omega$ is equicontinuous on $I$.

(b) In fact, when $t \rightarrow \infty$, we have

$$
\lim _{t \rightarrow \infty} \frac{G(t, s)}{1+t^{\alpha-1}}=\frac{1}{\Delta} \begin{cases}\lambda \eta^{2 \alpha-2}-\lambda(\eta-s)^{2 \alpha-2}, & s \leq \eta, \\ \lambda \eta^{2 \alpha-2}, & 0 \leq \eta \leq s .\end{cases}
$$

From this, it is not difficult to verify that for any given $\varepsilon>0$, there exists a constant $T^{\prime}=T^{\prime}(\varepsilon)>0$ such that

$$
\left|\frac{G\left(t_{1}, s\right)}{1+t_{1}^{\alpha-1}}-\frac{G\left(t_{2}, s\right)}{1+t_{2}^{\alpha-1}}\right|<\varepsilon
$$

for any $t_{1}, t_{2} \geq T^{\prime}$ and $s \in J$. Hence, $T$ is equiconvergent at $\infty$.

Thus the conclusion of Theorem 4 applies that $T$ is relatively compact on $J$. So, $T: X \rightarrow X$ is completely continuous. This completes the proof.

\section{Main Results}

Theorem 8. Assume that $\left(H_{1}\right)$ and $\left(H_{2}\right)$ with $\rho=1$ hold. If there exists $r>0$ such that

$$
r\left(1-L b^{*}\right)>L a^{*}
$$

with L given by (15), then problem (1) has a solution $u(t)$ satisfying

$$
0 \leq \frac{u(t)}{1+t^{\alpha-1}} \leq r, \quad \text { for } t \in J
$$

Proof. Let $U=\left\{u \in X,\|u\|_{X}<r\right\}$. For $u \in \partial U$, if there exist $v \in(0,1)$ such that $u=v T u$, then we have

$$
\begin{aligned}
\|u\|_{X} & =\sup _{t \in J}\left|\frac{v(T u)(t)}{1+t^{\alpha-1}}\right| \\
& \leq \sup _{t \in J} \int_{0}^{\infty} \frac{G(t, s)}{1+t^{\alpha-1}}|f(s, u(s))| d s \\
& \leq L \int_{0}^{\infty}|a(s)+b(s) u(s)| d s \\
& \leq L a^{*}+L b^{*}\|u\|_{X} .
\end{aligned}
$$

This implies that

$$
r\left(1-L b^{*}\right) \leq L a^{*}
$$

which contradicts (27). By Lemma 7 and Theorem 3, we conclude that problem (1) has a solution $u(t)$ satisfying

$$
0 \leq \frac{u(t)}{1+t^{\alpha-1}} \leq r, \quad t \in J
$$

This completes the proof.

In the next, we formulate existence results for the cases $0<\rho<1$ and $\rho>1$. We do not provide the proof of these results as it is similar to that of Theorem 8 . For that, we denote $\left(H_{2}\right)$ with $0<\rho<1$ and $\rho>1$, respectively, by $\left(H_{3}\right)$ and $\left(H_{4}\right)$.

Theorem 9. Let the assumptions $\left(H_{1}\right)$ and $\left(H_{3}\right)$ hold. Then problem (1) has a solution $u(t)$ satisfying

$$
0 \leq \frac{u(t)}{1+t^{\alpha-1}} \leq r, \quad t \in J
$$

where $r>\max \left\{2 L a^{*},\left(2 L b^{*}\right)^{1 /(1-\rho)}\right\}$ with L given by (15).

Theorem 10. Suppose that $\left(H_{1}\right)$ and $\left(H_{4}\right)$ hold and that there exists $2 L a^{*} \leq r \leq\left(2 L b^{*}\right)^{1 /(1-\rho)}$ with $L$ given by (15). Then problem (1) has a solution $u(t)$ such that

$$
0 \leq \frac{u(t)}{1+t^{\alpha-1}} \leq r, \quad t \in J
$$

\section{Example}

Example 1. With $\alpha=3 / 2, \lambda=1 / 2$, and $\eta=1$, we consider the following boundary value problem:

$$
\begin{gathered}
D^{3 / 2} u(t)+\frac{|u(t)|+\sin u(t)}{8\left(1+t^{1 / 2}\right)(1+t)^{2}}+\frac{4}{(t+4)^{2}}=0, \quad t \in[0,+\infty) \\
I^{1 / 2} u(0)=0 \\
D^{1 / 2} u(+\infty)=\frac{1}{2} I^{1 / 2} u(1) .
\end{gathered}
$$

Clearly the condition $\left(H_{1}\right)$ holds as $\Gamma(2 \alpha-1)=\Gamma(2)=1$, $\lambda \eta^{2 \alpha-2}=1 / 2$. Letting $a(t)=4 /(t+4)^{2}, b(t)=1 /\left(4\left(1+t^{1 / 2}\right)(1+\right.$ $t)^{2}$ ), we find that

$$
\begin{gathered}
f(t, u(t))=\frac{|u(t)|+\sin u(t)}{8\left(1+t^{1 / 2}\right)(1+t)^{2}}+\frac{4}{(t+4)^{2}} \\
\leq a(t)+b(t)|u(t)|, \\
\int_{0}^{+\infty} a(t) d t=1<+\infty, \\
\int_{0}^{+\infty}\left(1+t^{\alpha-1}\right) b(t) d t=\frac{1}{4}<+\infty .
\end{gathered}
$$

This shows that $\left(H_{2}\right)$ holds true. Finally, fixing $r>1 /(\sqrt{\pi}-$ 1 ), it can easily be verified that the condition (27) is satisfied. Thus all the conditions of Theorem 8 are satisfied. Therefore, by Theorem 8 , problem (27) has a solution $u(t)$ such that

$$
0 \leq \frac{u(t)}{1+t^{\alpha-1}} \leq r
$$




\section{Acknowledgments}

The work was supported by the Natural Science Foundation for Young Scientists of Shanxi Province, China (no. 2012021002-3). This paper was funded by King Abdulaziz University, under Grant no. (130-1-1433/HiCi). The authors, therefore, acknowledge technical and financial support of KAU. The authors also thank the reviewers for their useful comments.

\section{References}

[1] I. Podlubny, Fractional Differential Equations, Academic Press, San Diego, Calif, USA, 1999.

[2] G. M. Zaslavsky, Hamiltonian Chaos and Fractional Dynamics, Oxford University Press, Oxford, UK, 2008.

[3] R. L. Magin, Fractional Calculus in Bioengineering, Begell House, Redding, Conn, USA, 2006.

[4] A. A. Kilbas, H. M. Srivastava, and J. J. Trujillo, Theory and Applications of Fractional Differential Equations, vol. 204 of North-Holland Mathematics Studies, Elsevier Science B.V., Amsterdam, The Netherlands, 2006.

[5] J. Sabatier, O. P. Agrawal, and J. A. T. Machado, Eds., Advances in Fractional Calculus: Theoretical Developments and Applications in Physics and Engineering, Springer, Dordrecht, The Netherlands, 2007.

[6] D. Baleanu, K. Diethelm, E. Scalas, and J. J. Trujillo, Fractional Calculus Models and Numerical Methods, Series on Complexity, Nonlinearity and Chaos, World Scientific, Boston, Mass, USA, 2012.

[7] M. P. Lazarević and A. M. Spasić, "Finite-time stability analysis of fractional order time-delay systems: Gronwall's approach," Mathematical and Computer Modelling, vol. 49, no. 3-4, pp. 475481, 2009.

[8] J. D. Ramírez and A. S. Vatsala, "Monotone iterative technique for fractional differential equations with periodic boundary conditions," Opuscula Mathematica, vol. 29, no. 3, pp. 289-304, 2009.

[9] S. Zhang, "Existence results ofpositive solutions to boundary value problem for fractional differential equation," Positivity, vol. 13, no. 3, pp. 583-599, 2009.

[10] B. Ahmad and J. J. Nieto, "Existence of solutions for nonlocal boundary value problems of higher-order nonlinear fractional differential equations," Abstract and Applied Analysis, vol. 2009, Article ID 494720, 9 pages, 2009.

[11] Z. Wei, Q. Li, and J. Che, "Initial value problems for fractional differential equations involving Riemann-Liouville sequential fractional derivative," Journal of Mathematical Analysis and Applications, vol. 367, no. 1, pp. 260-272, 2010.

[12] B. Ahmad and S. K. Ntouyas, "A four-point nonlocal integral boundary value problem for fractional differential equations of arbitrary order," Electronic Journal of Qualitative Theory of Differential Equations, no. 22, pp. 1-15, 2011.

[13] Y. Zhao, S. Sun, Z. Han, and M. Zhang, "Positive solutions for boundary value problems of nonlinear fractional differential equations," Applied Mathematics and Computation, vol. 217, no. 16, pp. 6950-6958, 2011.

[14] B. Ahmad and J. J. Nieto, "Riemann-Liouville fractional integrodifferential equations with fractional nonlocal integral boundary conditions," Boundary Value Problems, vol. 2011, article 36, 2011.
[15] G. Wang, "Monotone iterative technique for boundary value problems of a nonlinear fractional differential equation with deviating arguments," Journal of Computational and Applied Mathematics, vol. 236, no. 9, pp. 2425-2430, 2012.

[16] G. Wang, D. Baleanu, and L. Zhang, "Monotone iterative method for a class of nonlinear fractional differential equations," Fractional Calculus and Applied Analysis, vol. 15, no. 2, pp. 244-252, 2012.

[17] A. Babakhani, "Positive solutions for system of nonlinear fractional differential equations in two dimensions with delay," Abstract and Applied Analysis, vol. 2010, Article ID 536317, 16 pages, 2010.

[18] V. Gafiychuk, B. Datsko, V. Meleshko, and D. Blackmore, "Analysis of the solutions of coupled nonlinear fractional reactiondiffusion equations," Chaos, Solitons \& Fractals, vol. 41, no. 3, pp. 1095-1104, 2009.

[19] A. Arara, M. Benchohra, N. Hamidi, and J. J. Nieto, "Fractional order differential equations on an unbounded domain," Nonlinear Analysis. Theory, Methods \& Applications A, vol. 72, no. 2, pp. 580-586, 2010.

[20] X. Zhao and W. Ge, "Unbounded solutions for a fractional boundary value problems on the infinite interval," Acta Applicandae Mathematicae, vol. 109, no. 2, pp. 495-505, 2010.

[21] S. Liang and J. Zhang, "Existence of three positive solutions of $m$-point boundary value problems for some nonlinear fractional differential equations on an infinite interval," Computers \& Mathematics with Applications, vol. 61, no. 11, pp. 3343-3354, 2011.

[22] X. Su, "Solutions to boundary value problem of fractional order on unbounded domains in a Banach space," Nonlinear Analysis. Theory, Methods \& Applications A, vol. 74, no. 8, pp. 2844-2852, 2011.

[23] S. Liang and J. Zhang, "Existence of multiple positive solutions for $m$-point fractional boundary value problems on an infinite interval," Mathematical and Computer Modelling, vol. 54, no. 56, pp. 1334-1346, 2011.

[24] G. Wang, B. Ahmad, and L. Zhang, "A coupled system of nonlinear fractional differential equations with multipoint fractional boundary conditions on an unbounded domain," Abstract and Applied Analysis, vol. 2012, Article ID 248709, 11 pages, 2012.

[25] Y. Liu, "Existence and uniqueness of solutions for initial value problems of multi-order fractional differential equations on the half lines," Scientia Sinica Mathematica, vol. 42, no. 7, pp. 735756, 2012.

[26] L. Zhang, B. Ahmad, G. Wang, and R. P. Agarwal, "Nonlinear fractional integro-differential equations on unbounded domains in a Banach space," Journal of Computational and Applied Mathematics, vol. 249, pp. 51-56, 2013.

[27] R. P. Agarwal, M. Meehan, and D. O’Regan, Fixed Point Theory and Applications, vol. 141, Cambridge University Press, Cambridge, UK, 2001.

[28] Y. Liu, "Existence and unboundedness of positive solutions for singular boundary value problems on half-line," Applied Mathematics and Computation, vol. 144, no. 2-3, pp. 543-556, 2003. 


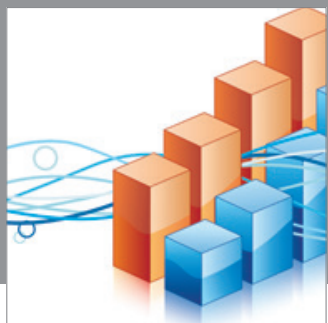

Advances in

Operations Research

mansans

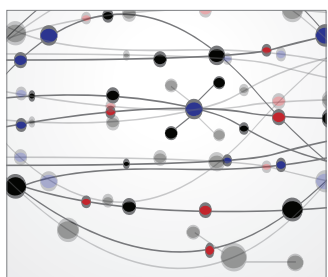

The Scientific World Journal
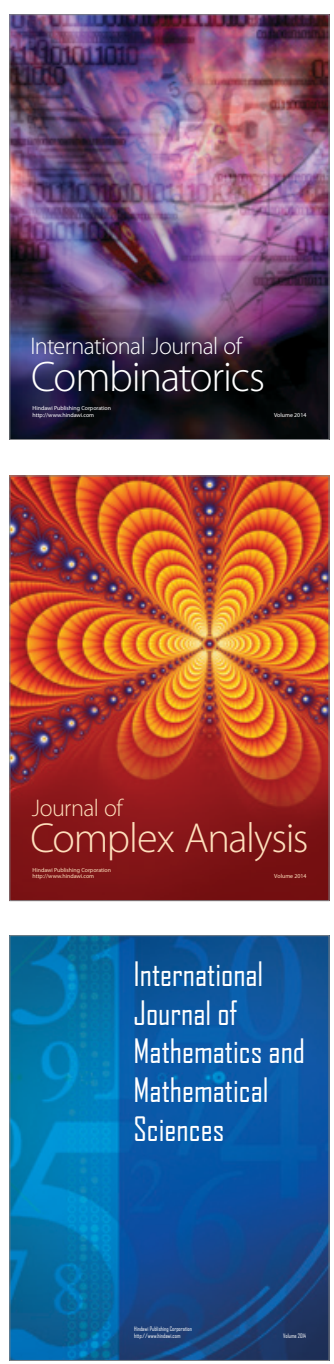
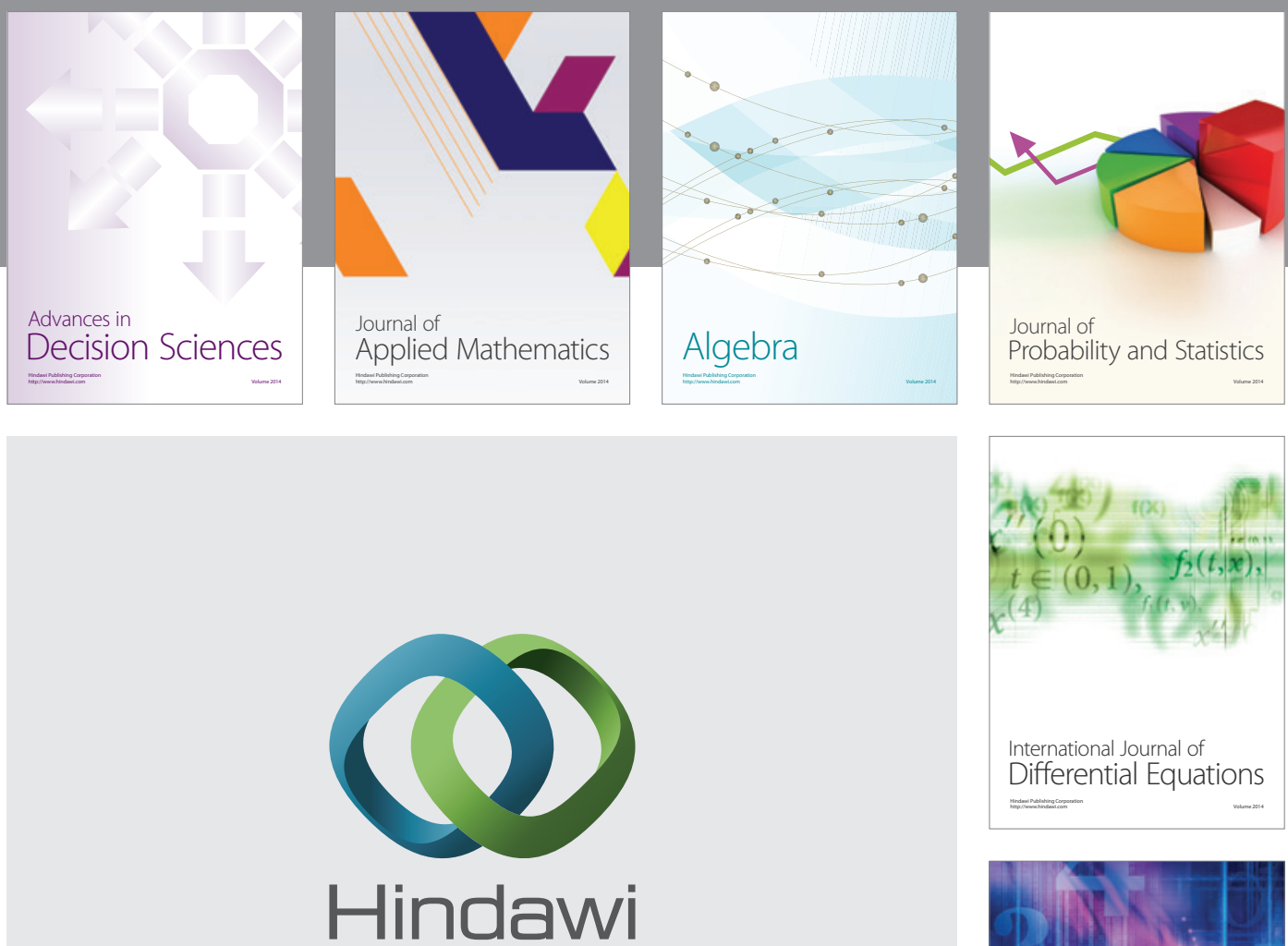

Submit your manuscripts at http://www.hindawi.com
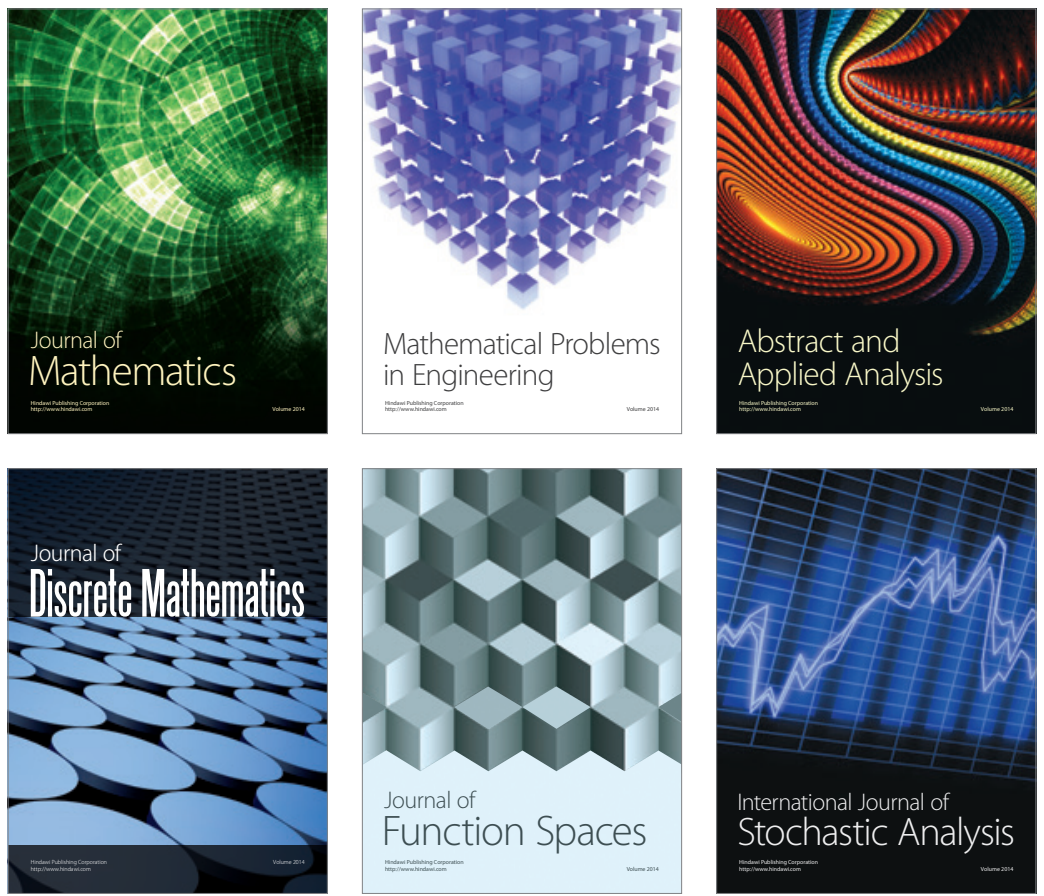

Journal of

Function Spaces

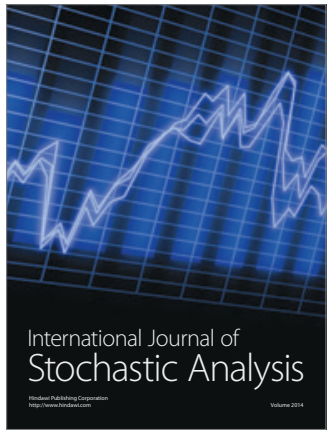

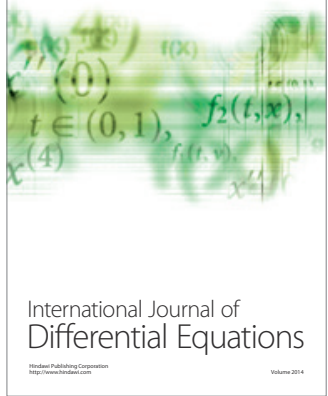
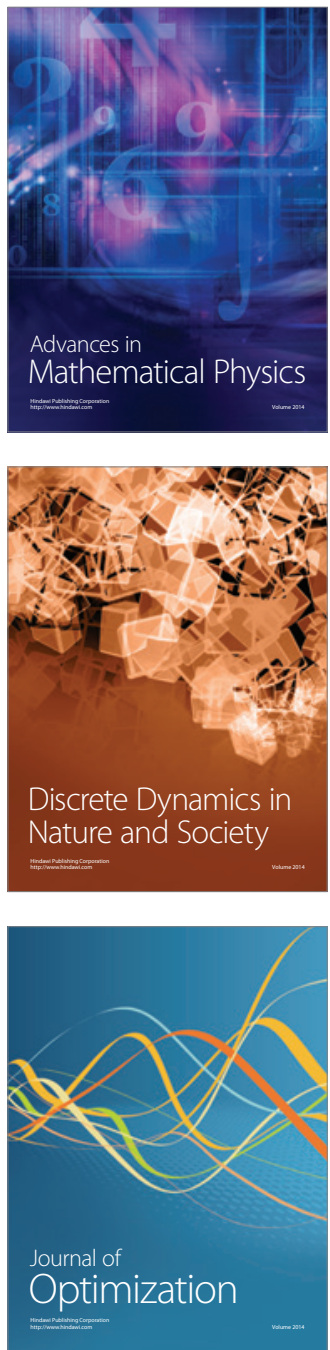